\title{
Tecnologia e educação em saúde: avaliação de um website para o ensino de oftalmologia
}

\section{Technology and education in health: evaluation of a website for the teaching of ophthalmology}

\begin{abstract}
Filipe Araújo Alves de Lima ${ }^{1}$ Francisca Tereza de Galiza $\bullet$ Ana Roberta Vilarouca da Silva ${ }^{\bullet}$ Eveline Pinheiro Beserra ${ }^{4}$ Jemima Rafaela Rodrigues de Medeiros ${ }^{5}$ - Maria Alzete de Lima 6
\end{abstract}

\begin{abstract}
RESUMO
Objetivo: Avaliar a tecnologia em saúde como estratégia de ensino em oftalmologia. Método: Estudo de avaliação de material educacional digital, desenvolvido na universidade pública no Piauí, de maio a dezembro de 2014 . Na primeira fase, foi elaborado um material digital para promoção da saúde ocular, seguido da submissão do material ao julgamento de 592 estudantes universitários. O estudo obteve aprovação do comitê de ética da instituição com número do parecer 508.069. Resultado: Foi possível identificar falhas na estruturação das páginas e os comandos tiveram de ser recolocados, unificados e dispostos em local de fácil visualização. O Website possuiu uma taxa de retorno ao site de 47,2\%; de acesso em dez países diferentes por jovens entre 20 e 24 anos, 66,7\%; de facilitação ao aprendizado, 56,1\%; de ser considerada uma forma interessante de promover saúde, 60,4\%. Quanto aos elementos estruturais, 70,4\% afirma ser de fácil compreensão, 51,9\% conseguiram identificar as estruturas oculares com facilidade, e 51,9\% conseguiram realizar o autoexame. Conclusão: A tecnologia facilitou a identificação de alterações visuais, mostrando-se eficaz no método de aprendizagem de prevenção em saúde ocular.
\end{abstract}

Palavras Chave: Saúde ocular; Enfermagem; Tecnologia biomédica.

\section{ABSTRACT}

Evaluating health technology as a strategy for teaching in ophthalmology. Method: Evaluation study of digital educational material developed in Piauí State University from May to December 2014. In the first phase was prepared digital material to promote eye health, followed by the submission of material to the trial of 592 students. The study obtained approval from the Ethics Committee of the institution with the number 508,069 opinion. Result: It was possible to identify flaws in the structure of the pages and the commands had to be reseated, unified and barranged in easy-to-see location. Website owned return rate to $47.2 \%$, accessed in ten different countries, between 20 to 24 years young, $66.7 \%$, the site facilitated learning, $56.1 \%$, and was considered an interesting way to promote health, $60.4 \%$. As for the structural elements, $70.4 \%$ claims to be easy to understand, 51.9 percent were able to identify the ocular structures with ease, and $51.9 \%$ were able to perform self-examination. Conclusion: The technology facilitated the identification of Visual changes, showing effective learning method of prevention in eye health.

Key words: Eye health; Nursing; Biomedical technology.

\footnotetext{
* Projeto financiado pelo CNPQ processo 443832/20145.

${ }^{1}$ Enfermeiro, graduação, Universidade Federal do Piauí, Picos, PI, Brasil. E-mail: lipepiaui@hotmail.com.

2 Enfermeira, mestre, Professora Universidade Federal do Piauí, Picos, PI, Brasil. E-mail: terezagaliza@yahoo.com.br

${ }^{3}$ Enfermeira, doutora, Professora da Universidade Federal do Piauí, Picos, PI, Brasil. E-mail: vilarouca@ufpi.edu.br.

${ }^{4}$ Enfermeira, doutora, Professor Universidade Federal do Ceará, Fortaleza, CE, Brasil. E-mail: eve_pinheiro@yahoo.com.br.

Enfermeira

${ }^{5}$ Acadêmica do Curso de Enfermagem da Universidade Federal do Rio Grande do Norte, Natal, RN, Brasil. E-mail: jemymarafaela@hotmail.com.

${ }^{6}$ Enfermeira, doutora, Professora Universidade Federal do Rio Grande do Norte, Natal, RN, Brasil. E-mail: alzetelima@ahoo.com.br.
} 


\section{INTRODUÇÃO}

Nos últimos anos, a educação no mundo vem sofrendo novas intervenções tecnológicas por proporcionar ferramentas e mídias digitais que ofertam ao processo pedagógico espaços e instrumentos capazes de renovar as situações de interação e comunicação, tornando-o diferente do modelo tradicional de ensino.

Um estudo realizado sobre a prevalência de utilização dos serviços de saúde ocular nos últimos cinco anos apontou que do total de pessoas entrevistadas, $34 \%$ não realizaram consulta oftalmológica. Entre os motivos, destacaram-se baixas condições financeiras e indisponibilidade de tempo $0^{1-2}$.

Logo, capacitar as pessoas ao autoexame favorece o autocuidado, pois muitas alterações oculares podem ser identificadas quando há o conhecimento prévio sobre saúde ocular. Assim, a educação da população continua a ser uma ferramenta vital na prevenção e gestão adequada de uma injúria ocular ${ }^{3}$.

$\mathrm{Na}$ promoção da saúde ocular, há programas para avaliação da acuidade visual que buscam identificar indivíduos com significativos erros de refração, executando ações essenciais para o controle da deficiência visual. Entretanto, as ações de promoção que antecipam a identificação precoce de mudanças nas características oculares e visuais são muitas vezes negligenciadas ${ }^{4}$.

Verifica-se, portanto, que as ações educativas constroem a base para a promoção da saúde ocular, constituindose como condição necessária e antecedente às ações do indivíduo para preservar a visão, aumentando a capacidade de tomar decisões relativas a comportamentos que influenciarão seu nível de saúde ${ }^{5}$.

Corrobora-se com a afirmação de que ao se oportunizar o acesso a materiais educativos, com apoio nas tecnologias disponíveis, estimula-se o aprendiz à mudança de comportamento na medida em que o torna sujeito do processo de aprendizagem ${ }^{6}$. Essa afirmação toma dimensões ampliadas quando se combinam ações de educação, autocuidado e de prevenção em saúde.

Portanto, a busca por alternativas que capacitem as pessoas a realizarem avaliação ocular periódica torna-se um método auxiliar no processo de promoção da saúde ocular. Os meios de comunicação têm potencial de atingir amplo número de pessoas, a um custo menor do que o método de intervenção individual. Isso foi recentemente comprovado por uma pesquisa que utilizou a escala de Snellen e instruções sobre sua utilização em quatro jornais diários. Por meio da pesquisa telefônica com 603 pessoas, assinantes destes jornais, descobriu-se que 125 utilizaram a escala para testar a sua visão ${ }^{7}$.

$\mathrm{Na}$ perspectiva de associar medidas de prevenção em saúde voltada à estratégias de educação em saúde e aproveitando-se das novas possibilidades na área da tecnologia da informação e comunicação, buscou-se avaliar tecnologia em saúde como estratégia de ensino em oftalmologia.

\section{METODOLOGIA}

Estudo de avaliação de material educacional virtual ${ }^{6}$, desenvolvido em uma universidade pública no interior do Brasil, de maio a dezembro de 2014. As etapas do estudo consistiram de: definição dos objetivos de aprendizagem, elaboração de um website sobre saúde ocular, intitulado Enfermagem Oftalmológica, avaliação e usabilidade do material. Quanto à elaboração do material instrucional, realizou-se um planejamento e definições acerca das mídias utilizadas, vídeos, imagem e hipertexto, da estruturação das ferramentas a serem disponibilizadas e das formas de feedback ao usuário.

Após a elaboração do material, este foi enviado para o endereço de e-mail de 240 estudantes universitários do curso de Enfermagem. Considerando o objeto do estudo, foi determinado como critério de inclusão estudantes do quinto semestre, por já terem cursado semiologia adotado para evitar viés de coleta e com experiência no ambiente da Internet. Recrutados por meio de envio de e-mail pelo Sistema Integrado de Gestão de Atividades Acadêmicas, apenas 27 responderam com assinatura do Termo de Consentimento, no qual se declaravam possuir elegíveis a participar do estudo e acessaram o Website, onde, por meio de um link, puderam conhecer o material educativo digital. Depois da leitura do material, o usuário tinha acesso a um questionário online que versava sobre o resultado do exame ocular e questões sobre características do uso e organização do site. Era solicitado preenchimento do instrumento, encorajando-se o envio de sugestões.

Os questionários tratavam do propósito de utilização do objeto educacional, organização, adequação da tecnologia aos sujeitos, sua aparência e usabilidade.De posse dos instrumentos preenchidos e com as sugestões dos participantes, seguiu-se as reformulações do objeto educacional.

Todas estas etapas seguiram recomendações de estudos recentes, nos quais se destaca que a fase de construção de material educacional digital deve ser implementada de forma cíclica, ou seja, perfazendo o caminho de construção, teste e avaliação ${ }^{6,8}$.

0 acesso ao site http://autoocularsiteufpi.wixsite. com/autoocularsiteufpi foi computado por ferramenta do Google Analytics que tem capacidade de possibilitar em números absolutos todos os tipos de acesso do site, e os instrumentos reenviados foram digitados semanalmente pelo pesquisador em programa Excel. Para o relato das sugestões aceitas, seguiu-se codificação A1, A2... A27 para identificar falas dos acadêmicos.

O site versa sobre autocuidado visual e informações sobre a anatomia, fisiologia e semiologia da visão, além de assegurar a realização do autoexame ocular, há um link de acesso a informação de como realizar esse autoexame, que consiste em um conhecimento básico das partes internas e externas dos olhos, seu funcionamento e avaliação da acuidade visual longe/perto, das estruturas oculares, movimento ocular, visão periférica e central. A avaliação da acuidade visual é medida com o auxílio de escala de Snellen, amplamente utilizada em avaliações oftalmológicas. 
O site foi estruturado de forma simples e rápida, seguindo uma ordem a partir da anatomia para se conhecer primeiramente as estruturas oculares e suas respectivas funções e o conjunto sensorial constituído pelo olho, via óptica, centros visuais e um conjunto não sensorial representado pelos vasos e nervos.

O estudo foi realizado conforme a Resolução 466/12 da Comissão Nacional de Ética em Pesquisa, obtendo aprovação pelo comitê de ética da Universidade Federal do Piauí sob nº 508.069.

\section{RESULTADOS}

Buscou-se desenvolver material educativo em website para promoção, prevenção e autocuidado ocular em mídia digital para uso em ambiente virtual. A intenção é permitir à população conhecer informações sobre a visão e orientar sobre o autoexame ocular, para assim, ampliar a efetiva promoção da saúde ocular. Segundo a Figura 1, o website possuiu 597 sessões, com total de 592 usuários, 647 visualizações de páginas.

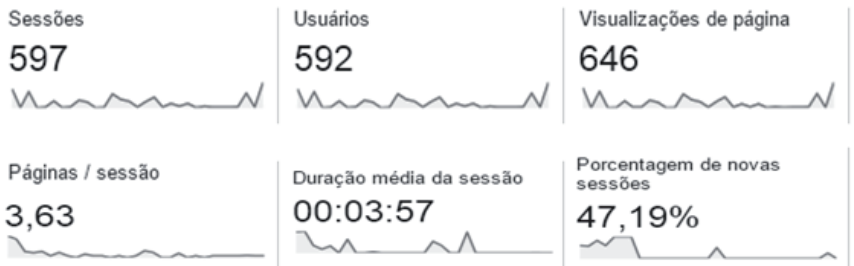

Figura 1: Informações de frequência de aceso do web site, Piauí, Brasil, 2014.

Fonte: Dados da pesquisa.

A etapa de teste sobre o uso do material educacional foi importante para se diagnosticar falhas na elaboração. Alguns itens foram reformulados, como tornar os comandos mais visíveis ao usuário, facilidade de troca de páginas para guiar melhor o seguimento de navegação, e troca de ilustrações de modo a tornar sua compreensão mais clara. Com isso, cumpriram-se importantes itens avaliativos do material educacional para acesso à distância.

O site apresentou um percentual de $47,2 \%$ de novos visitantes e um retorno de $52,8 \%$, o qual comprova que o site oferece informações pertinentes para proporcionar 0 desejo de voltar a acessar o conteúdo.

Quanto ao país de origem, a maioria dos acessos foi no Brasil, 519(32,3\%), isso se justifica pela realização do estudo no país. E reitera-se, ainda que não se tenha realizado a tradução do site, registrou-se $165(27,6 \%)$ acessos nos Estados Unidos, 37(6,2\%) na China e 32(5,3\%) Japão. Em diferentes proporções obteve-se acesso de dez diferentes países. Entretanto, de acordo com os critérios elegibilidade dos participantes do estudo, selecionou-se dentre os usuários os dados referentes aos estudantes que estavam cursando semiologia do curso de enfermagem.

\begin{tabular}{|c|c|}
\hline Pais & 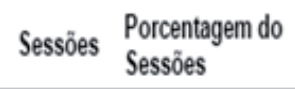 \\
\hline 1. 图 Brazil & $193 \square 32,33 \%$ \\
\hline 2. 国 United States & $165 \square 27,64 \%$ \\
\hline 3. China & 37 | $6,20 \%$ \\
\hline 4. - Japan & 32 | $5,36 \%$ \\
\hline 5. 回 Germany & $24 \mid 4,02 \%$ \\
\hline 6. (not set) & $16 \mid 2,68 \%$ \\
\hline 7. South Korea & $14 \mid 2,35 \%$ \\
\hline 8. $=$ Netherlands & $10 \mid 1,68 \%$ \\
\hline 9. [I] Canada & $6 \mid 1,01 \%$ \\
\hline 10. & $6 \mid 1,01 \%$ \\
\hline
\end{tabular}

Figura 2: Frequência de acesso, segundo país de origem, Piauí, Brasil, 2014.

Fonte: Dados da pesquisa.

Tabela 1. Frequência de respostas segundo dados sobre usabilidade do website, conhecimento sobre saúde ocular e local de acesso. Piauí, Brasil, 2014.

\begin{tabular}{|c|c|c|}
\hline Variável $^{*}$ & $\mathrm{n}$ & $\%$ \\
\hline \multicolumn{3}{|l|}{ Faixa etária } \\
\hline Menos de 20 anos & 1 & 3,7 \\
\hline 20 a 24 anos & 18 & 66,7 \\
\hline 25 a 30 anos & 4 & 14,8 \\
\hline Acima de 30 anos & 4 & 14,8 \\
\hline \multicolumn{3}{|l|}{ Conhecimento sobre saúde ocular } \\
\hline Desconhece & 7 & 20,6 \\
\hline Aponta alguma doença ocular & 12 & 35,3 \\
\hline Declara saber avaliar saúde ocular & 7 & 20,6 \\
\hline Sente-se seguro para realizar autoexame ocular & 8 & 23,5 \\
\hline \multicolumn{3}{|l|}{ Interesse na web site } \\
\hline Facilidade de entendimento & 23 & 56,1 \\
\hline $\begin{array}{l}\text { Acesso à informação digital é mais atrativo que } \\
\text { os livros }\end{array}$ & 18 & 43,9 \\
\hline \multicolumn{3}{|l|}{ Potencializa a aprendizagem } \\
\hline Vídeo & 25 & 45 \\
\hline Figura & 11 & 19,5 \\
\hline Hiperlink de acesso a pagina & 7 & 12,4 \\
\hline Fórum & 12 & 21,3 \\
\hline Outros & 1 & 1,8 \\
\hline \multicolumn{3}{|l|}{0 que potencializa aprendizagem no web site } \\
\hline $\begin{array}{l}\text { Por ser uma forma interessante de promoção da } \\
\text { saúde }\end{array}$ & 26 & 60,4 \\
\hline A cartilha sobre autoexame ocular & 11 & 25,6 \\
\hline Apresenta efeito gráfico chamativo & 5 & 11,7 \\
\hline Outros & 1 & 2,3 \\
\hline
\end{tabular}

Fonte: Dados da pesquisa.

* com exceção da idade, considerou-se mais de uma alternativa como resposta. 
Os participantes do estudo eram jovens com idade entre 20 e 24 anos, 18 (66,7\%). Sobre o conhecimento em oftalmologia, chama atenção o fato de que 7 (20,6\%) desconheciam o tema, ainda assim, 12 (35,3\%) conseguiram apontar algum tipo de doença ocular e 8 (23,5\%) conseguiam realizar com segurança e corretamente 0 autoexame ocular.

Quanto aos recursos disponíveis, 23(56,1\%) afirmaram que 0 site facilita o entendimento sobre a realização correta do exame ocular, $18(43,9 \%)$ declararam que o acesso a informação virtual é mais atrativa que os livros convencionais.

Em relação a melhor forma de aprendizado, os internautas elegeram os vídeos, $25(45 \%)$, como a forma de aprendizagem mais fácil e $12(21,3 \%)$ escolheram o fórum. Grande parte dos participantes considerou o website como ferramenta importante de promoção da saúde, 26(60,4\%). Entre os componentes do site de maior importância $11(25,6 \%)$ elegeram a cartilha sobre o autoexame ocular; e $5(11,7 \%)$ referiram à disposição gráfica do site.

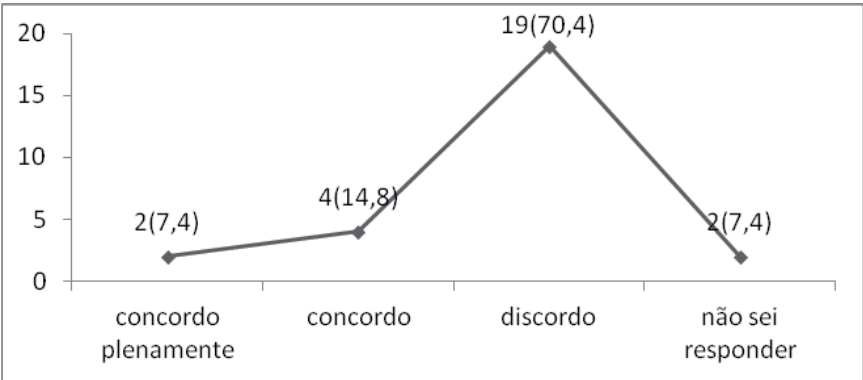

Figura 3: Frequências de respostas obtidas segundo existência de termos técnicos que dificultam a aprendizagem, Piauí, Brasil, 2014.

Fonte: Dados da pesquisa.

A respeito da compreensão textual, 19 (70,4\%) discordam com a afirmação de haver dificuldade de entendimento da linguagem em decorrência de termos técnicos e 02 (7,4\%) não souberam responder se havia dificuldade em identificar as estruturas oculares, como se observa na figura 4.

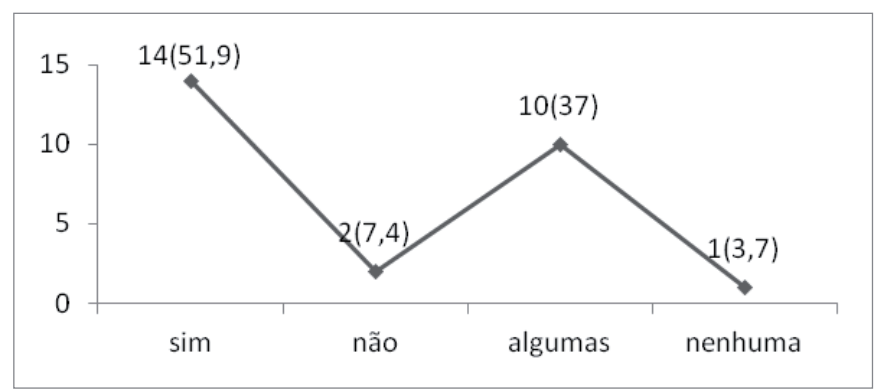

Figura 4: Frequências de respostas obtidas segundo capacidade de identificar as estruturas oculares, Piauí, Brasil, 2014.

Fonte: Dados da pesquisa.

Quanto à observância de aprendizado e identificação das estruturas oculares, 14(51,9\%) usuários afirmaram que após visitar o site e acessar as informações sentiamse seguros em realizar exame e identificar as estruturas oculares, enquanto que $10(37 \%)$ concordavam ter dificuldade no exame.

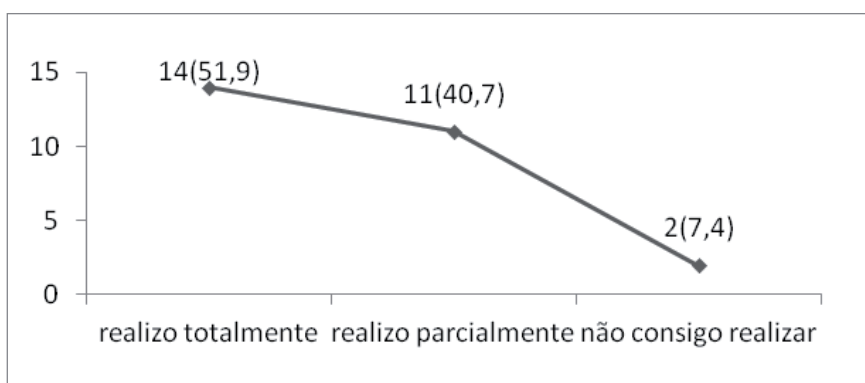

Figura 5: Frequências de respostas obtidas segundo capacidade de realizar do autoexame ocular, Piauí, Brasil, 2014.

Fonte: Dados da pesquisa.

Sobre sua realização, figura 6, 14(51,9\%) afirmaram ser possível concluir o autoexame, já 11(40,7\%) dizem conseguir realizar parcialmente e dois dos usuários relataram não conseguir realizar o exame ocular sozinho, o que corrobora a necessidade de se abordar esse assunto no site.

Nas avaliações emergiram sugestões e opiniões sobre o material, destacando-se a adequação da linguagem ao público alvo e a estratégia de reduzir os textos. $\mathrm{Na}$ concepção dos alunos e professores envolvidos, o material se encontra adequado para o público alvo e caracterizase como um instrumento a ser utilizado na educação do público leigo.

Sugiro que durante as instruções tenham figuras autoexplicativas [...] A1

Adequar o vocabulário. Não que ele esteja muito técnico, mas o ideal é que ele seja mais detalhado [...]A2 Senti falta das fontes de onde as informações e imagens foram retiradas, bem como as devidas referências. Sugiro uma revisão ortográfica para melhorar a redação A3

Após a análise das avaliações sugeridas pelos acadêmicos, considerando ter-se questionado sobre os termos técnicos, foi implementada apreciação especialista na área de letras, para melhor adequar a linguagem e avaliar aspectos relacionados à clareza e redução do texto.

\section{DISCUSSÃO}

É inegável a complexidade de uma assistência integral relativa aos problemas oculares, porém, é preciso considerar que se pode trabalhar com um público interessado em saúde ocular utilizando instrumentos capazes de atingir amplo número de pessoas, de modo a sensibilizá-las a incorporarem a prática do autoexame no seu cotidiano. Justificando-se tal esforço, ao considerar que alguns estudos apontam desconhecimento do público alvo sobre as medidas de prevenção sobre saúde ocular̊. 
Pesquisadores corroboram com a ideia de que a aprendizagem on-line requer mudança qualitativa na natureza da comunicação e interação ${ }^{10-11}$. O desafio atualmente centra-se na busca por materiais que auxiliem no processo educacional do público de forma geral, na aquisição de conhecimento e tomada de decisão dos usuários dos serviços de saúde.

Notadamente, grupos de pesquisadores vêm investigando as melhores práticas na educação on-line na Enfermagem, considerando-se o uso da tecnologia, as práticas educacionais, o suporte ao educando e os resultados ${ }^{12}$. Comprova-se que há uma correlação entre a prática educativa on-line e a satisfação do usuário, no sentido de tornar o processo mais autônomo.

A respeito da adequação do material educativo digital às características do usuário, os menus possibilitam que se navegue livremente em uma estrutura linear e flexível. Acredita-se que a possibilidade do usuário ir para o exame que deseja realizar aumenta seu interesse e estimula o aprendizado ${ }^{13}$.

Pode-se observar que o público jovem prevaleceu entre os usuários, a maioria conseguiu realizar o autoexame ocular com segurança, assim se observou que o material educativo auxiliou na promoção do autocuidado ocular.

Destaca-se que o material educacional digital caracteriza-se por conteúdos didáticos com emprego de multimídia e interatividade associado a recursos das tecnologias da informática e da comunicação. Esses recursos digitais são elaborados seguindo um planejamento integrado ao processo de aprendizagem, delineados dentro de uma perspectiva pedagógica ${ }^{11}$.

Entretanto, se busca atingir padrões de layout de forma a reduzir a carga cognitiva funcional, porque cada componente necessitará ser percebido e interpretado pelo aprendiz. Uma tela que use convenções padrão no texto, gráficos, navegação e layout simples terá os seus componentes mais facilmente interpretados e consequentemente terá uma carga cognitiva mais baixa ${ }^{14}$.

Comprovadamente os novos paradigmas pedagógicos, mediados pelo surgimento das novas tecnologias, propõem desafios aos profissionais envolvidos no processo educacional. Corrobora-se com a ideia de que o uso desses novos recursos extrapolam a visão tradicional e os métodos meramente discursivos no processo de ensino aprendizagem ${ }^{14}$. Comprovadamente 0 site se mostrou eficiente para auxiliar no autoexame, pois $23,5 \%$ dos visitantes afirmam conseguir realizar avaliação ocular.

Estudos recentes mostram que um material bem elaborado e uma informação de fácil entendimento melhoram o conhecimento e a satisfação do paciente, desenvolvem ações que influenciam o padrão de saúde e favorecem a tomada de decisão, além de contribuir na redução do uso dos serviços e dos custos com a saúde ${ }^{15}$.

Sua incorporação no processo de cuidado em saúde reverte-se comprovadamente em resultados clínicos positivos ${ }^{16}$. Assim como no processo de autocuidado ${ }^{17}$, o controle de sintomas por meio do telemonitoramento domiciliar ${ }^{18}$ aumenta a qualidade de vida e a doença crônica ${ }^{19}$ reduz as taxas de readmissão tempo de internações, custos em saúde, promove segurança do paciente, melhora a independência na realização de atividades de vida diária e adesão à terapêutica ${ }^{20}$.

O feedback recebido deste estudo motivou a melhorar o material e buscar estratégias de maior divulgação e distribuição, cuja intenção é realizar um estudo com amplo número de usuários, e a importância da realização de cada etapa pode proporcionar maior eficácia na construção de um material educativo digital.

Por isso que os objetos de aprendizagem se configuram tanto como ferramentas para apoiar a educação à distância, como ferramentas complementares na construção e fixação de novos conceitos.

Destaca-se que esta tecnologia virtual tem o caráter de oferecer informação de forma sistemática, ou seja, possui as características de acessibilidade e reutilização, como observado que o número de retorno de visitantes; supera o número de novos visitantes. Portanto, espera-se que o acesso de forma ilimitada proporcione cada vez mais a vontade e o desejo de conhecimento da saúde.

\section{CONCLUSÃO}

Verificou-se que materiais educativos na área de enfermagem instigam a vontade de aprender, e esse tipo de aprendizagem cumpre com as necessidades dos usuários no uso das novas tecnologias, inseridas atualmente no cotidiano das pessoas.

Para melhor orientar o usuário, a página inicial da web site foi reestruturada direcionando os usuários aos links de autoexame ocular, fórum de discussão e ao aprendizado das funções e alterações visuais.

A habilidade que o profissional enfermeiro possui em desenvolver tecnologias e trabalhar com novas ferramentas em prol da promoção da saúde, entendendo que o computador e a Internet fazem parte do cotidiano de uma grande parcela da população.

Portanto, é fundamental o uso de novas tecnologias do cuidado com enfoque nas ações educativas, entretanto, é necessário uma avaliação de suas limitações, benefícios e uma adequação às necessidades dos usuários; já que a avaliação é um processo contínuo de aperfeiçoamentos e ajustes mais adequados; e assim propor um caminho inovador que gere atitudes conscientes e intencionais, além da valorização e reconhecimento do exercício de cidadania.

O website pode ser utilizado como estratégia complementar no processo de aprendizagem, já que facilita a identificação de alterações visuais precoces, e também proporcione incentivo na busca de uma assistência oftalmológica. 


\section{REFERÊNCIAS}

1. Sousa RP, Miota FMCSC, Carvalho ABG. Tecnologias digitais na educação. Campina Grande: EDUEPB, 2011. 276p.

2. Castagno VD, Fassa AG, Silva MC, Carret MLV. Carência de atenção à saúde ocular no setor público: um estudo de base populacional. Cad Saúde Pública. 2009;25(10):2260-72.

3. Okpala NE, Umeh RE, Onwasigwe EN. Eye Injuries Among Primary School Children in Enugu, Nigeria: Rural vs Urban. Ophthalmology and Eye Diseases. 2015;7:13-19.

4. Lazard A, Mackert M. User evaluations of design complexity: The impact of visual perceptions for effective online health communication. International Journal of Medical Informatics, 2014; 83(10):726-735.

5. Temporini ER, Kara-José N. Níveis de prevenção de problemas oftalmológicos. Arq Bras Oftalmol. 1995;58(3):189-92.

6. Falkembach GAM. Concepção e desenvolvimento de material educativo digital. Novas Tecnologias na Educação. 2005;3(1):1-15.

7. Murray E, Burns J, See TS, Lai R, Nazareth I. Interactive health communication applications for people with chronic disease. Cochrane Database Syst Rev. 2005;19(4):CD004274.

8. Behar PA, Passerino L, Bernardi M. Modelo pedagógico para a educação a distância: pressupostos teóricos para a construção de objetos de aprendizagem. Novas Tecnologias. 2007;5(2).

9. Grady JL. The Virtual Clinical Practicum: an innovative telehealth model for clinical nursing education. Nurs Educ Perspect. 2011;32(3):189-94.

10. Tarouco LMR, Grando ARCS, Konrath ML. Alfabetização visual para a produção de objetos educacionais. CINTED-UFRGS, Porto Alegre. 2003;1(2).

11. Gracindo HBR, Fireman EC. Laboratório de informática, os objetos digitais de aprendizagem e a visão do professor. Educação à Distância e Práticas Educativas Comunicacionais e Interculturais. 2010;4(4).

12. Tanaka RY, Catalan VM, Zemiack J, Pedro ENR, Cogo ANP, Silveira DT. Objeto educacional digital: avaliação da ferramenta para prática de ensino em enfermagem. Acta Paul Enferm 2010;23(5):603-7.

13. Fonseca LMM, Leite AM, Vasconcelos MGL, Castral TC, Scochi, CGS. Cartilha educativa on line sobre os cuidados com o bebê pré-termo: aceitação dos usuários. Ciência Cuidado e Saúde. 2007;6(2).

14. Tarouco LMR, Cunha, SLS. A aplicação de teorias cognitivas ao projeto de objetos de aprendizagem. CINTED-UFRGS Novas Tecnologias da Educação. 2006;4(2).

15. Oliveira MS, Fernandes AFC, Sawada NO. Manual educativo da mulher mastectomizada: um estudo de validação. Texto Contex Enferm. 2008;17(1):115-123.

16. Gardner LA, Sparnon EM. Work-Arounds Slow Electronic Health Record Use. American Journal of Nursing. 2014;114(4):64-67.

17. Lingley-Pottie P, McGrath PJ, Andreou P. Barriers to mental health care: perceived delivery system differences. Adv Nurs Sci. 2013;36(1):51-61.

18. Minatodani DE, Chao PJ, Berman SJ. Home telehealth: facilitators, barriers, and impact of nurse support among highrisk dialysis patients. Telemed J E Health. 2013;19(8):573-8.
19. Brennan PF, Casper GR, Burke ப, Johnson KA, Brown $\mathrm{R}$, Valdez RS, et al. Technology-enhanced practice for patients with chronic cardiac disease: home implementation and evaluation. Heart \& Lung. 2010;39(6):S34-S46.

20. Gardner LA, Sparnon EM. Work-Arounds Slow Electronic Health Record Use. American Journal of Nursing. 2014;114(4):64-67. 